\title{
Exact Solutions of the Dirac Hamiltonian on the Sphere under Hyperbolic Magnetic Fields
}

\author{
Özlem Yeşiltaş \\ Department of Physics, Faculty of Science, Gazi University, 06500 Ankara, Turkey \\ Correspondence should be addressed to Özlem Yeşiltaş; yesiltas@gazi.edu.tr
}

Received 5 April 2014; Accepted 11 June 2014; Published 1 July 2014

Academic Editor: Filipe R. Joaquim

Copyright @ 2014 Özlem Yeşiltaş. This is an open access article distributed under the Creative Commons Attribution License, which permits unrestricted use, distribution, and reproduction in any medium, provided the original work is properly cited. The publication of this article was funded by $\mathrm{SCOAP}^{3}$.

\begin{abstract}
Two-dimensional massless Dirac Hamiltonian under the influence of hyperbolic magnetic fields is mentioned in curved space. Using a spherical surface parameterization, the Dirac operator on the sphere is presented and the system is given as two supersymmetric partner Hamiltonians which coincides with the position dependent mass Hamiltonians. We introduce two ansatzes for the component of the vector potential to acquire effective solvable models, which are Rosen-Morse II potential and the model given Midya and Roy, whose bound states are Jacobi $X_{1}$ type polynomials, and we adapt our work to these special models under some parameter restrictions. The energy spectrum and the eigenvectors are found for Rosen-Morse II potential. On the other hand, complete solutions are given for the second system. The vector and the effective potentials with their eigenvalues are sketched for each system.
\end{abstract}

\section{Introduction}

Since the isolation of graphene investigated in 2004 by Novoselov et al. [1], this new material has spurred an intense interest in its applications in the fields of the condensed matter physics and the quantum field theory. The charge carriers in flat graphene are modeled in continuum by the Dirac-Weyl operator for massless fermions [2,3]. Apart from some spectacular properties of graphene such as the halfinteger quantum Hall effect $[1,2,4-6]$, observation of Klein tunneling for the two-dimensional massless Dirac electrons [7-9], algebraic approaches to impurities [10], and graphene wormholes [11], interesting flexural modes of the graphene have also attracted much attention [12-17]. According to the Landau-Peierls theorem, the flat graphene does not exist at all $[18,19]$. The curvature effects on the quality factor of the graphene nanoresonators can be found in [20]. Moreover, it is seen that graphene nanoribbons can be the solution of improving energy storage in ultra-high capacitors and bending nanodevices [21-23]. In this manner, instead of a flat graphene, curved graphene has such mechanical and electrical properties that lead to the possibility of a bending component of some nanodevices. In the relativistic territory, the Dirac equation in curved spacetime has been studied as its generalization to the Robertson-Walker spacetime in a Cartesian tetrad gauge [24], the gravitational effects in Hydrogen atom [25], bound states of the Dirac equation in gravitational fields [26], exact solutions in $(1+1)$ and $(2+1)$ dimensions $[27,28]$, some conditions on the modified Dirac equation which admits a symmetry operator [29], and the Hawking-Unruh phenomenon on graphene [30]. On the other hand, the system on the two-sphere or on the pseudosphere is related to some interesting generalizations of the planar Landau level problems [31,32]. The supersymmetry is known to be responsible for the mathematical structure of the Landau levels. The Pauli Hamiltonian for a nonrelativistic spin- $1 / 2$ particle possesses $N=2$ supersymmetry $[33,34]$. The Dirac equation and its analysis and discussion within supersymmetric quantum mechanics can be found in [3539]. Moreover, extending a study of Bochner [40], exceptional orthogonal polynomials were introduced first in [41]. Later, it is shown that there is a relation between exceptional orthogonal polynomials and the Darboux transformation $[42,43]$. 
In this study, the Dirac equation in curved space is written for the curved graphene models to show that an exactly solvable bound state model for the Dirac equation in a twodimensional curved space can be reduced into a Schrödingerlike operator and the bound states can be discussed using the concepts of quantum mechanics. Rosen-Morse II potential (hyperbolic Rosen-Morse) model is studied as a first example and the next one is related with the soliton models [44]. This potential family is empirically useful to investigate the polyatomic vibrational states of $\mathrm{NH}_{3}$ molecule [45]. Also, trigonometric Rosen-Morse potential is studied in the confinement phenomena of quarks in hadrons and this potential is responsible for the quark-gluon dynamics through the QCD calculations [46, 47]. Hyperbolic potentials are popular in nonrelativistic quantum mechanics with their soliton models [48]. Considering these instanton structures, hyperbolic interactions and their connection with the QCD and Morse-Rosen potentials can be found in an interesting work [49]. Also, actual relativistic vibrational states for the molecules are studied in [50]. Thus, our study may be a motivation in the relativistic domain for the relevant QCD models.

\section{Dirac Operator in Two-Dimensional Curved Space}

On a two-dimensional surface, the Dirac operator is given by [51]

$$
\mathscr{H}_{D}=-i \gamma^{a}\left(E_{a}^{\mu} \partial_{\mu}+\frac{1}{2} E_{a}^{\mu} \partial_{\mu} \ln \sqrt{g}+\frac{1}{2} \partial_{\mu} E_{a}^{\mu}\right) .
$$

The symbol $\gamma^{\mu}$ corresponds to the generalized Dirac matrices satisfying the relation

$$
\gamma^{a} E_{a}^{\mu}=\gamma^{\mu}
$$

and the anticommutation relation is

$$
\left\{\gamma^{\mu}, \gamma^{\nu}\right\}=2 g^{\mu \nu}
$$

Here, $E_{a}^{\mu}$ are the tetrad fields, $g$ is the determinant of the metric tensor, and $g_{\mu \nu}$ is the metric tensor for the curved spacetime; the Latin indices refer to local inertial frame and the Greek indices refer to curved spacetime. Generally, $g_{\mu \nu}$ is given by

$$
g_{\mu \nu}(\mathbf{x})=\eta_{a b} e_{\mu}^{a}(\mathbf{x}) e_{\nu}^{b}(\mathbf{x}),
$$

where $\mathbf{x}=(t, x), \eta_{a b}=\operatorname{diag}(+1,-1)$, and $e_{\mu}^{a}$ and $E_{a}^{\mu}$ are zweibeins and their inverse satisfies

$$
\delta^{a b} E_{a}^{\mu} E_{b}^{\nu}=g^{\mu \nu}, \quad g_{\mu \nu} E_{a}^{\mu} E_{b}^{v}=\delta_{a b} .
$$

The conformal metric tensor is given by [51]

$$
g_{\mu \nu}=e^{2 \sigma}\left(\begin{array}{ll}
1 & 0 \\
0 & 1
\end{array}\right),
$$

where $\sigma(\vec{x})$ is a spatial function. Using (5) and (6), we obtain $E_{a}^{\nu}=e^{-\sigma} \delta_{a}^{\nu}$. As it is explained in [51], $P_{\mu}=-i \partial_{\mu}$ is the flat momentum operator replaced by $P_{\mu} \rightarrow P_{\mu}-A_{\mu}$, where $A_{\mu}$ is the vector potential which is spatial dependent. In two dimensions, Dirac matrices reduce to Pauli matrices; then, the Dirac operator becomes

$$
\mathscr{H}_{D}=-2 i e^{\sigma}\left(\begin{array}{cc}
0 & \partial_{z}-i A_{z}+\frac{1}{2} \partial_{z} \sigma \\
\partial_{z^{*}}-i A_{z^{*}}+\frac{1}{2} \partial_{z^{*}} \sigma & 0
\end{array}\right),
$$

where ${ }^{*}$ denotes the complex conjugate and the transformation $z=x^{1}+i x^{2}$ is used in the Hamiltonian above for the sake of simplicity. In this manner, we use $\partial_{z}=(1 / 2)\left(\partial_{1}-i \partial_{2}\right)$, $A_{z}=(1 / 2)\left(A_{1}-i A_{2}\right)$. The operator (7) is also studied in [52]. Now we take $\mathscr{H}_{D}$ which can be mapped to the operator $\overline{\mathscr{H}}_{D}$ using a transformation operator $U$ as

$$
\overline{\mathscr{H}}_{D}=U \mathscr{H}_{D} U^{-1}, \quad U=\left(\begin{array}{cc}
e^{\sigma / 2} & 0 \\
0 & e^{\sigma / 2}
\end{array}\right) .
$$

Therefore, we have

$$
\overline{\mathscr{H}}_{D}=\alpha\left(\begin{array}{cc}
0 & \partial_{1}-i \partial_{2}-i A_{1}-A_{2} \\
\partial_{1}+i \partial_{2}-i A_{1}+A_{2} & 0
\end{array}\right),
$$

where $\alpha=-i e^{\sigma}$. Let us bring the attention to the isothermal coordinates and parameterization [53]. Because the topological equivalent of a layer is known as a sphere, then we may deal with a sphere with radii $R$ and the surface parameterization of the sphere is given by

$$
\mathbf{x}(u, v)=R(\cos u \cos v, \sin u \cos v, \sin v),
$$

and the metric can be written as

$$
\begin{aligned}
(d s)^{2} & =R^{2}\left(\cos ^{2} v d u^{2}+d v^{2}\right) \\
& =R^{2} \cos ^{2} v\left(d u+i \frac{d v}{\cos v}\right)\left(d u-i \frac{d v}{\cos v}\right) .
\end{aligned}
$$

We may rewrite the metric using the transformation $w=$ $\log (\tan v+\sec v)$. Then, the metric of the patch is written as

$$
d s^{2}=\lambda^{2}\left(d u^{2}+d w^{2}\right),
$$

where $\lambda=R \cos v$. If we use the transformation $\cosh w=$ $\sec v$, we get $e^{\sigma}=R \operatorname{sech} w$. It can be pointed out that the eigenvectors of the operator $\overline{\mathscr{H}}_{D}$ are $\Psi=e^{i k u}\left(\begin{array}{c}\psi_{1} \\ i \psi_{2}\end{array}\right)$, where we take $x^{1}=w$ and $x^{2}=u$, and $k$ is the wave number. We also note that the vector potential and its components are selected as $A_{\mu}=\left[0, A_{\mathcal{u}}(w), 0\right]$. Let us now look at the eigenvalue equation $\overline{\mathscr{H}}_{D} \Psi(\vec{x})=E \Psi(\vec{x})$.

\section{Dirac Hamiltonians}

The Dirac Hamiltonian in (9) can be rewritten as

$$
\begin{aligned}
& \alpha\left(\partial_{w}-i \partial_{u}-A_{u}(w)\right)\left(i e^{i k u} \psi_{2}(w)\right)=E\left(e^{i k u} \psi_{1}(w)\right), \\
& \alpha\left(\partial_{w}+i \partial_{u}-A_{u}(w)\right)\left(e^{i k u} \psi_{1}(w)\right)=E\left(i e^{i k u} \psi_{2}(w)\right) .
\end{aligned}
$$


The equations given above lead to

$$
\mathscr{H}_{D}^{j} \psi_{j}(w)=\bar{E}^{2} \psi_{j}(w), \quad \bar{E}=E R,
$$

where $j=1,2$. Then, Hamiltonians, $\mathscr{H}_{D}^{j}$, shown in a joint expression can be given as

$$
\begin{aligned}
\mathscr{H}_{D}^{j}= & -\cosh ^{2} w \frac{d^{2}}{d w^{2}}-\sinh w \cosh w \frac{d}{d w} \\
+ & {\left[\left(\left(k-A_{u}(w)\right)^{2}+(-1)^{j} A^{\prime}(w)\right) \cosh ^{2} w\right.} \\
& \left.+(-1)^{j}\left(A_{u}(w)-k\right) \cosh w \sinh w\right] .
\end{aligned}
$$

We may transform (14) into a Sturm-Liouville type equation using a similarity transformation. We obtain

$$
\begin{aligned}
\mathfrak{h}_{j} & =\rho^{-1} \overline{\mathscr{H}}_{D}^{j} \rho, \\
\rho & =\sqrt{\cosh w} \Longrightarrow \mathfrak{h}_{j} \\
& =-\cosh ^{2} w \frac{d^{2}}{d w^{2}}-2 \sinh w \cosh w \frac{d}{d w}+V_{\text {eff }}^{j}(w),
\end{aligned}
$$

where

$$
\begin{aligned}
V_{\mathrm{eff}}^{j}(w)= & \left(\left(k-A_{u}(w)\right)^{2}+(-1)^{j} A^{\prime}(w)\right) \cosh ^{2} w \\
& +(-1)^{j}\left(A_{u}(w)-k\right) \cosh w \sinh w \\
& -\frac{3}{4} \cosh ^{2} w+\frac{1}{4}
\end{aligned}
$$

Thus, the transformed Dirac system can be given as

$$
\mathfrak{h}_{j} \phi_{j}=\bar{E}^{2} \phi_{j}, \quad \phi_{j}=\rho \psi_{j}
$$

We emphasize that the solutions $\phi_{j, n}$ have to satisfy $\int_{\Omega}\left\{\left|\phi_{1, n}(w)\right|^{2}+\left|\phi_{2, n}(w)\right|^{2}\right\} d w<\infty$ for the physical reasons, and here $n$ is a quantum number. We will examine (18) in the following section using the choices of $A_{u}(w)$.

3.1. Hyperbolic Model I. Let us choose $A_{u}(w)$ as

$$
A_{u}(w)=C_{1} \operatorname{sech}^{2} w+C_{2} \tanh w+C_{3},
$$

where $C_{1}, C_{2}, C_{3}$ are constants; we may obtain

$$
\begin{aligned}
\bar{V}_{\mathrm{eff}}^{1}(w) \\
=-C_{2}+2 C_{1} C_{3}-2 C_{1} k \\
\quad+\left(\left(C_{3}-k\right)^{2}-\frac{1}{2}\right) \cosh ^{2} w+C_{1}^{2} \operatorname{sech}^{2} w \\
\quad+\left(-C_{3}+2 C_{2} C_{3}+k-2 C_{2} k\right) \cosh w \sinh w \\
\quad+\left(C_{2}^{2}-C_{2}-\frac{1}{4}\right) \sinh ^{2} w+C_{1}\left(1+2 C_{2}\right) \tanh w
\end{aligned}
$$

Equating the coefficients of $\cosh w \sinh w, \cosh ^{2} w$, and $\sinh ^{2} w$ to zero, one can obtain $C_{2}$ and $C_{3}$ as

$$
\left\{\left(C_{2}\right) ;\left(C_{3}\right)\right\}=\left\{\left(-\frac{1}{2}, \frac{1}{2}, \frac{1}{2}, \frac{3}{2}\right) ;(k,-1+k, 1+k, k)\right\}
$$

Thus, (20) becomes

$$
\begin{aligned}
\bar{V}_{\text {eff }}^{1}(w)= & C_{1}^{2} \operatorname{sech}^{2} w+C_{1}\left(1+2 C_{2}\right) \tanh w \\
& +\left(C_{2}-\frac{1}{2}\right)^{2}+2 C_{1}\left(C_{3}-k\right)-\frac{1}{2}
\end{aligned}
$$

and also $V_{\mathrm{eff}}^{2}(w)$ turns into

$$
\begin{aligned}
\bar{V}_{\mathrm{eff}}^{2}(w)= & C_{1}^{2} \operatorname{sech}^{2} w+C_{1}\left(-1+2 C_{2}\right) \tanh w \\
& +2 C_{2} \cosh ^{2} w+2\left(C_{3}-k\right) \cosh w \sinh w \\
& +\left(C_{2}+\frac{1}{2}\right)^{2}-2 C_{1}\left(C_{3}-k\right)-\frac{1}{2} .
\end{aligned}
$$

As it is seen from (23), $V_{\text {eff }}^{2}(w)$ is not a solvable potential model while (22) is known as Rosen-Morse II potential [54]. As a result, they are not shape invariant potentials but they share the same energy spectrum except the ground state. If we take $C_{2} \neq-1 / 2$, this implies $C_{3}=k-1, C_{3}=k+1$, or $C_{3}=k$. Next, we will come up with the whole solutions of the system (22). Hence, we use the transformations given in (18)

$$
\phi_{1}=\operatorname{sech}^{2} w G(t), \quad t=\tanh w
$$

and we get

$$
\begin{aligned}
\frac{d^{2} G}{d t^{2}}- & \frac{4 t}{1-t^{2}} \frac{d G}{d t}+\frac{1}{\left(1-t^{2}\right)^{2}} \\
& \times\left(\bar{E}^{2}-\left(C_{1}^{2}+2\right)\left(1-t^{2}\right)-C_{1}\left(1+2 C_{2} t\right)\right. \\
& \left.\quad-\left(C_{2}-\frac{1}{2}\right)^{2}-2 C_{1}\left(C_{3}-k\right)+\frac{1}{2}\right) G(t)=0 .
\end{aligned}
$$

We propose a solution which is $G(t)=(1-t)^{(a-1) / 2}(1+$ $t)^{(b-1) / 2} F(t)$, where $F(t)$ is a polynomial that we look for and we obtain

$$
\begin{gathered}
\left(1-t^{2}\right) \frac{d^{2} F}{d t^{2}}+(b-a-(a+b+2) t) \frac{d F}{d t} \\
+\frac{1}{1-t^{2}}\left(\mathbf{A} t^{2}+\mathbf{B} t+\mathbf{C}\right) F(t)=0
\end{gathered}
$$


where $\mathbf{A}, \mathbf{B}, \mathbf{C}$ are constants. Hence, the solutions of the system are given as

$$
\begin{aligned}
E_{1, n}= \pm \frac{1}{R}\left(\frac{1}{2}+2 C_{1}\left(k-C_{3}\right)-\left(C_{2}-\frac{1}{2}\right)^{2}\right. & \\
& -\left(\frac{-1+\sqrt{1-4 C_{1}^{2}}}{2}-n\right)^{2} \\
& \left.-\frac{\left(C_{1}\left(1+2 C_{2}\right) / 2\right)^{2}}{\left(\left(-1+\sqrt{1-4 C_{1}^{2}}\right) / 2-n\right)^{2}}\right)^{1 / 2} \\
\phi_{1, n}= & N_{1}(1-\tanh w)^{\left(-1+\sqrt{1-4 C_{1}^{2}}\right) / 2}(1+\tanh w)^{C_{1}\left(1+2 C_{2}\right) / 2} \\
& \times P_{n}^{\left(-1+\sqrt{1-4 C_{1}^{2}}, C_{1}\left(1+2 C_{2}\right)\right)}(\tanh w)
\end{aligned}
$$

where the symbol $P_{n}^{(\cdot, \cdot)}(\tanh w)$ corresponds to the Jacobi polynomials. We also note that $C_{1}<1 / 2$ for the physical solutions. It is noted that $\bar{V}_{\text {eff }}^{2}(w)$ which is not a solvable model shares the same spectrum with $\bar{V}_{\text {eff }}^{1}(w)$ except for the ground state [54] (Figure 1).

\subsection{Hyperbolic Model II. If we set $A_{u}$ as given below}

$$
A_{u}(w)=C_{1} \operatorname{sech}^{2} w+C_{2} \frac{\operatorname{sech}^{2} \tanh w}{a_{1} \tanh w-a_{2}}+C_{3} \tanh w+C_{4},
$$

where we use constants $C_{1}, C_{2}, C_{3}, C_{4}$, we may get

$$
\begin{aligned}
\bar{V}_{\mathrm{eff}}^{1}(w)= & \frac{1}{4}-C_{3}+2 C_{1} C_{4}-2 C_{1} k \\
& +\left(-\frac{3}{4}+\left(k-C_{4}\right)^{2}\right) \cosh ^{2} w+C_{1}^{2} \operatorname{sech}^{2} w \\
& +C_{3}\left(C_{3}-1\right) \sinh ^{2} w \\
& +\left(k-C_{4}+2 C_{3} C_{4}-2 C_{3} k\right) \cosh w \sinh w \\
& +C_{1}\left(1+2 C_{3}\right) \tanh w-\frac{C_{2} \operatorname{sech}^{2} w}{a_{1} \tanh ^{2} w} \\
& +\frac{a_{1} C_{2} \operatorname{sech}^{2} w \tanh w}{\left(a_{1} \tanh w-a_{2}\right)^{2}}+\frac{C_{2}^{2} \operatorname{sech}^{2} w \tanh ^{2} w}{\left(a_{1} \tanh ^{2} w a_{2}\right)^{2}} \\
& +\frac{2 C_{2}\left(C_{4}-k\right) \tanh w}{a_{1} \tanh w-a_{2}}+\frac{2 C_{1} C_{2} \operatorname{sech}^{2} w \tanh w}{\left(a_{1} \tanh ^{2} w-a_{2}\right)} \\
& +\frac{C_{2}\left(1+2 C_{3}\right) \tanh ^{2} w}{a_{1} \tanh w-a_{2}} .
\end{aligned}
$$

In [44], it is shown that a class of exactly solvable potentials, whose solutions correspond to the Jacobi-type $X_{1}$ exceptional orthogonal polynomials, can be generated. In their work, the eigenvalue equation is given by [44]

$$
H_{\mathrm{eff}} \psi(x)=\varepsilon \psi(x), \quad H_{\mathrm{eff}}=-\Delta+v_{\mathrm{eff}}(x),
$$

where $\psi(x)=f(x) F(g(x))$ and $F(g(x))$ satisfies the differential equation which is

$$
\frac{d^{2} F}{d g^{2}}+Q(g) \frac{d F}{d g}+R(g) F=0
$$

The authors choose $F_{n} \sim P_{n}^{(\alpha, \beta)}(x), n=1,2,3, \ldots, \alpha, \beta>$ $-1, \alpha \neq \beta$, where $P_{n}^{(\alpha, \beta)}(x)$ is the Jacobi-type $X_{1}$ polynomial in Example 2 in their work and obtain

$$
\begin{aligned}
\varepsilon- & v_{\mathrm{eff}}(x) \\
=\frac{g^{\prime}}{\lambda}\left(\frac{A_{1} g+A_{2}}{1-g^{2}}+\frac{A_{3} g+A_{4}}{\left(1-g^{2}\right)^{2}}\right. & \\
& \left.+\frac{A_{5}}{(\beta-\alpha) g-(\beta+\alpha)}+\frac{A_{6}}{((\beta-\alpha) g-(\beta+\alpha))^{2}}\right),
\end{aligned}
$$

where $A_{i}, i=1,2, \ldots, 6$ are constants in terms of $\alpha, \beta$ [44] and $g(x)=\tanh x$ and $\lambda$ is a constant. If $g(x)$ is used in (33), then we get

$$
\begin{aligned}
\varepsilon- & v_{\mathrm{eff}}(x) \\
= & \frac{A_{6} \operatorname{sech}^{2} x}{\left(a_{1} \tanh x-a_{2}\right)^{2}}+\frac{A_{5} \operatorname{sech} x}{\left(a_{1} \tanh x-a_{2}\right)} \\
& +A_{4} \cosh ^{2} x+A_{3} \sinh x \cosh x+A_{2}+A_{1} \tanh x .
\end{aligned}
$$

If we compare (30) and (33) we see that $\lambda=1, a_{1}=$ $\beta-\alpha$, and $a_{2}=\beta+\alpha$ in our problem. The energy spectrum and the solutions of (31) are given in [44]. Hence, we continue to compare $\varepsilon-v_{\text {eff }}(x)$ and $\bar{E}^{2}-\bar{V}_{\text {eff }}^{1}(w)$ which may provide complete solutions of our system without solving a differential equation. Let us add and subtract the terms $C_{5} \operatorname{sech}^{2} w /(-a 2+a 1 \tanh w)+C_{6}$ to the right-hand side of (30). Then, we shall obtain 


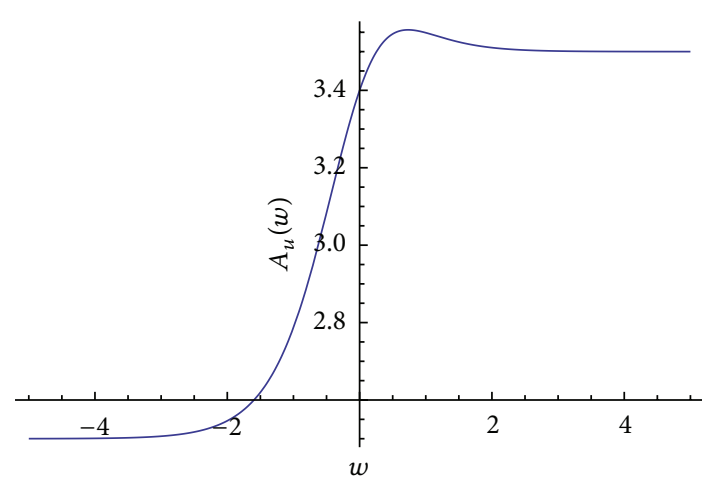

(a)

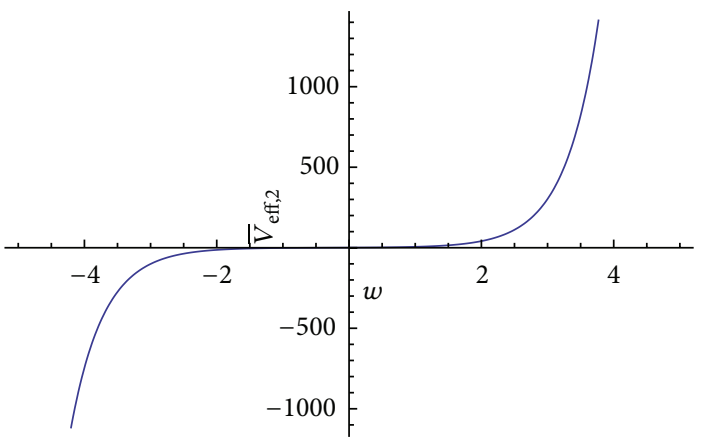

(c)

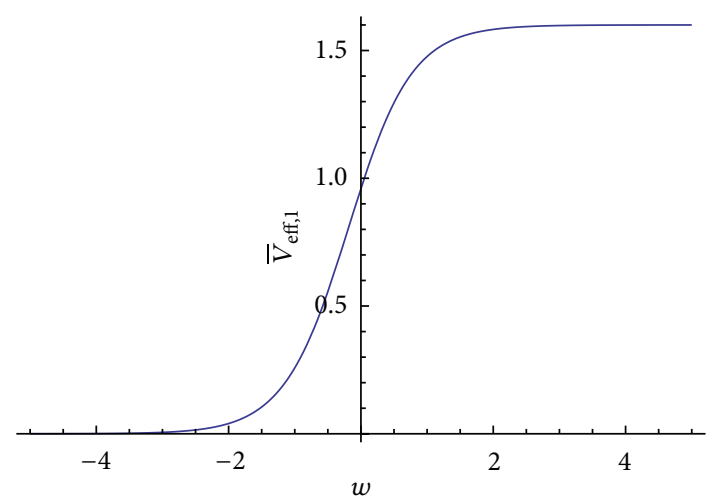

(b)

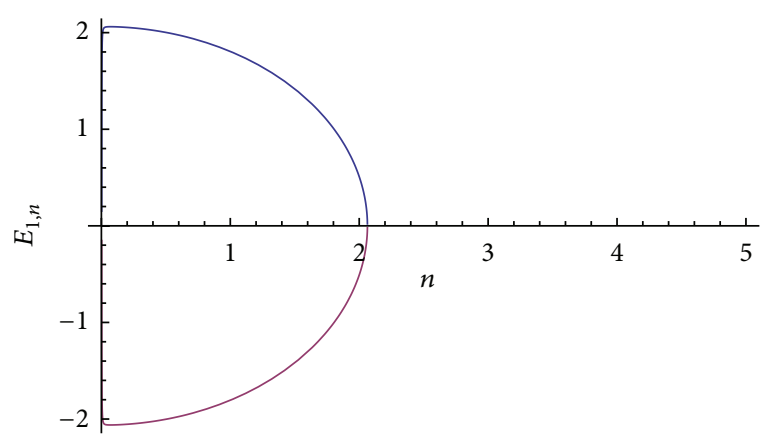

(d)

Figure 1: The graphs of (a) component of the vector potential $A_{u}(w)$ in (19), effective models (b) $\bar{V}_{\text {eff,1 }}$ (22) and (c) $\bar{V}_{\text {eff,2 }}$ (23) for the values of $C_{1}=0.4, C_{2}=0.5, R=1, C_{3}=k+1$, and $k=2$, and (d) the energies $\pm E_{1, n}$ in (27) for $k=200$. There are bound states only for $n=0,1,2$ and $\bar{V}_{\text {eff, } 2}$ is an increasing function in the positive domain while $\bar{V}_{\text {eff, } 1}$ remains nearly constant. The scalar component of the vector potential $A_{u}$ is similar to $\bar{V}_{\mathrm{eff}, 1}$.

$$
\begin{aligned}
\bar{V}_{\mathrm{eff}}^{1}(w)= & \frac{1}{4}-C_{6}+2 C_{1} C_{4}-2 C_{1} k-C_{3}^{2} \\
& +\left(\left(k-C_{4}\right)^{2}+\left(C_{3}-\frac{1}{2}\right)^{2}-1\right) \cosh ^{2} w \\
& +\left(k-C_{4}+2 C_{3} C_{4}-2 C_{3} k\right) \cosh w \sinh w \\
& +C_{1}\left(1+2 C_{3}\right) \tanh w \\
& -\frac{\left(C_{2}+C_{5}\right) \operatorname{sech}^{2} w}{a_{1} \tanh w-a_{2}}+\frac{\left(a_{2}^{2} C_{1}^{2}-a_{1} a_{2} C_{1}\right) \operatorname{sech}^{2} w}{\left(a_{1} \tanh w-a_{2}\right)^{2}}
\end{aligned}
$$

$$
\begin{aligned}
\bar{V}_{\mathrm{eff}}^{2}(w)= & \frac{1}{4}-C_{3}+2 C_{1} C_{4}-2 C_{1} k \\
& +\left(\left(k-C_{4}\right)^{2}-\frac{3}{4}\right) \cosh ^{2} w+C_{3}\left(1+C_{3}\right) \sinh ^{2} w \\
& +\left(C_{4}-k+2 C_{3} C_{4}-2 C_{3} k\right) \cosh w \sinh w \\
& +C_{1}\left(-1+2 C_{3}\right) \tanh w-\frac{a_{1} C_{1} \operatorname{sech}^{2} w}{a_{1} \tanh w-a_{2}}
\end{aligned}
$$

Here we may use some constraints on the parameters which may be expressed in terms of $C_{1}$,

$$
\begin{gathered}
C_{2}=-a_{1} C_{1}, \quad C_{3}=-\frac{a_{1}^{2}-a_{2}^{2}-2 a_{1} a_{2} k}{2\left(a_{1}^{2}-a_{2}^{2}\right)}, \\
C_{4}=-\frac{a_{2}^{2} k}{a_{1}^{2}-a_{2}^{2}}, \quad C_{5}=-2 a_{1} C_{1} C_{3}, \\
C_{6}=\frac{2 a_{1}^{2} k C_{1}}{a_{1}^{2}-a_{2}^{2}} .
\end{gathered}
$$




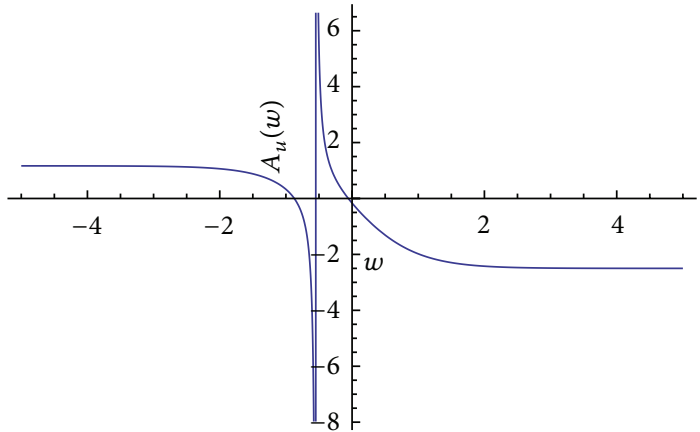

(a)

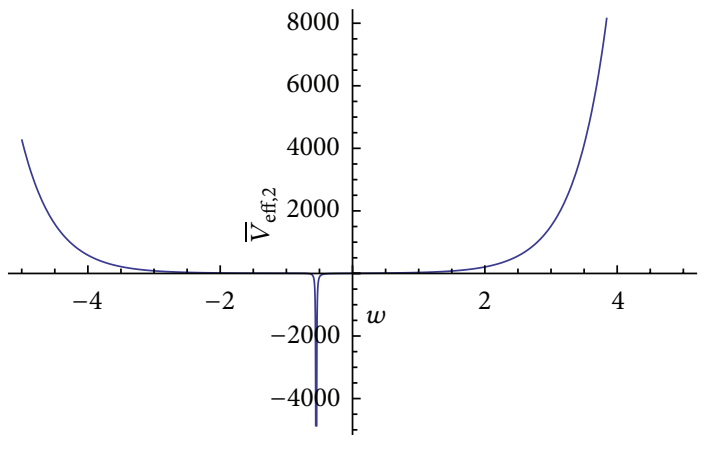

(c)

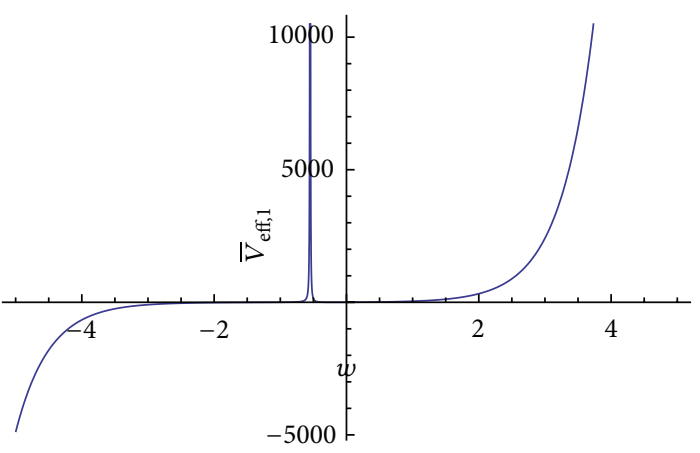

(b)

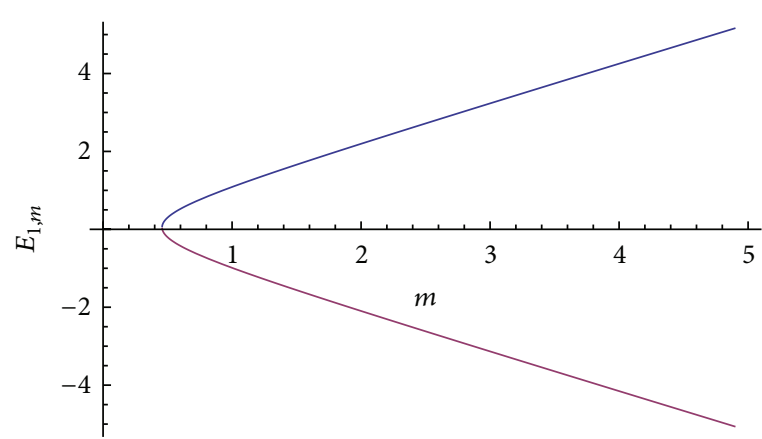

(d)

FIGURE 2: The graphs of (a) component of the vector potential $A_{u}(w)$ in (29), effective models (b) $\bar{V}_{\text {eff,1 }}$ (35) and (c) $\bar{V}_{\text {eff,2 }}$ (36), and (d) the energy $E_{1, n}$ in (40). The effective potential well $\bar{V}_{\mathrm{eff}, 2}$ and $\bar{V}_{\mathrm{eff}, 1}$, and $A_{u}(w)$ have the same singularity point. Energy eigenvalues are increasing.

Under these constraints, $\bar{V}_{\text {eff }}^{1}$ can be obtained as given in (35). Now we can compare (34) and (35) using $A_{i}, i=1,2, \ldots, 6$ given in [44]. These constants are given as [44]

$$
\begin{aligned}
A_{1}= & \frac{\beta^{2}-\alpha^{2}}{2 \alpha \beta}, \\
A_{2}= & n^{2}+(\beta+\alpha-1) n \\
& +\frac{1}{4}\left((\beta+\alpha)^{2}-2(\beta+\alpha)-4\right)+\frac{\beta^{2}+\alpha^{2}}{2 \alpha \beta}, \\
A_{3}= & \frac{\beta^{2}-\alpha^{2}}{2}, \quad A_{4}=-\frac{\beta^{2}+\alpha^{2}-2}{2}, \\
A_{5}= & \frac{(\beta+\alpha)(\beta-\alpha)^{2}}{2 \alpha \beta}, \quad A_{6}=-2(\beta-\alpha)^{2} .
\end{aligned}
$$

Using (34), (35), (37), and (38), we obtain

$$
\beta= \pm \frac{1}{1+k}, \quad \alpha= \pm \frac{1}{1-k}
$$

The energy eigenvalues and corresponding wave functions of the system are given in [44]. Using the results of [44] we may give the spectrum of our system as

$$
\begin{aligned}
E_{1, m}= \pm \frac{1}{R}( & \left(m+\frac{\alpha+\beta}{2}\right)\left(m+\frac{\alpha+\beta+2}{2}\right) \\
+ & \left.\frac{\beta}{\alpha}-\frac{\alpha^{2}+\beta^{2}-2}{4}-\frac{k^{2}}{\left(1+k^{2}\right)^{2}}\right)^{1 / 2},
\end{aligned}
$$

where $m=n-1, m=0,1,2, \ldots$ and one can see that the inside of the square root is positive. And the solutions are given by [44]

$$
\begin{aligned}
\phi_{1, m}(w)= & N_{m} \frac{(1-\tanh w)^{(\alpha+1) / 2}(1+\tanh w)^{(\beta+1) / 2}}{\alpha+\beta+(\alpha-\beta) \tanh w} \\
& \times P_{m+1}^{(\alpha, \beta)}(\tanh w),
\end{aligned}
$$

where $N_{m}$ is the normalization constant. Hence, $\bar{V}_{\text {eff }}^{2}(w)$ also shares the same energy except for the ground state [54] (Figure 2),

$$
E_{1, m}=E_{2, m-1}, \quad m=1,2, \ldots
$$




\section{Conclusion}

In this paper, we have studied the quantum behavior of a massless two-dimensional Dirac particle subjected to a hyperbolic magnetic field in the curved space which is a twodimensional surface on the sphere. Using appropriate transformations, we have given the Dirac Hamiltonian system a pair of first order differential equations and, then, effective Dirac Hamiltonians are expressed. Two specific models are studied according to the special choices of $A_{\mathcal{u}}(w)$. In fact, $\mathfrak{h}_{j}$ is the physical Hamiltonian of the system with a coefficient of the second order derivative, and $\cosh ^{2} w$ corresponds to the inverse of the mass function in the position dependent mass Hamiltonian theories. We have seen that this specific choice leads to two specific exactly solvable effective potential models. In the first example, the Rosen-Morse II effective potential is obtained under some parameter restrictions. Energy eigenvalues and corresponding eigenfunctions are obtained using polynomial solutions. In the latter case we have adapted our model Hamiltonian $\mathfrak{h}_{j}$ to a general one given in Example 2 in [44] and the bound state solutions are written. The partner potential $\bar{V}_{\text {eff }}^{2}$ is different and more general than those obtained in [44]. On the contrary to the results in [44], the partner potentials are not shape invariant in our case. We also note that we have obtained only a limited number of bound states for the Rosen-Morse II effective potential and when the radius of the sphere approaches infinity, the energy of the system becomes zero in each case.

\section{Conflict of Interests}

The author declares that there is no conflict of interests regarding the publication of this paper.

\section{References}

[1] K. S. Novoselov, A. K. Geim, S. V. Morozov et al., "Electric field in atomically thin carbon films," Science, vol. 306, no. 5696, pp. 666-669, 2004.

[2] K. S. Novoselov, A. K. Geim, S. V. Morozov et al., "Twodimensional gas of massless Dirac fermions in graphene," Nature, vol. 438, no. 7065, pp. 197-200, 2005.

[3] V. M. Pereira and A. H. Castro Neto, "Strain engineering of Graphene's electronic structure," Physical Review Letters, vol. 103, no. 4, Article ID 046801, 2009.

[4] Y. Zhang, J. P. Small, M. E. S. Amori, and P. Kim, "Electric field modulation of galvanomagnetic properties of mesoscopic graphite," Physical Review Letters, vol. 94, Article ID 176803, 2005.

[5] B. Huard, J. A. Sulpizio, N. Stander, K. Todd, B. Yang, and D. Goldhaber-Gordon, "Transport measurements across a tunable potential barrier in graphene," Physical Review Letters, vol. 98, Article ID 236803, 2007.

[6] R. V. Gorbachev, A. S. Mayorov, A. K. Savchenko, D. W. Horsell, and F. Guinea, "Conductance of p-n-p graphene structures with "air-bridge" top gates," Nano Letters, vol. 8, no. 7, pp. 1995-1999, 2008.

[7] N. Stander, B. Huard, and D. Goldhaber-Gordon, "Evidence for Klein tunneling in graphene $p-n$ junctions," Physical Review Letters, vol. 102, Article ID 026807, 2009.
[8] A. F. Young and P. Kim, "Quantum interference and Klein tunnelling in graphene heterojunctions," Nature Physics, vol. 5, pp. 222-226, 2009.

[9] P. E. Allain and J. N. Fuchs, "Klein tunneling in graphene: optics with massless electrons," European Physical Journal B, vol. 83, no. 3, pp. 301-317, 2011.

[10] T. Maier and H. Siedentop, "Stability of impurities with Coulomb potential in graphene with homogeneous magnetic field," Journal of Mathematical Physics, vol. 53, no. 9, Article ID 095207, 2012.

[11] J. Gonzalez and J. Herrero, "Graphene wormholes: a condensed matter illustration of Dirac fermions in curved space," Nuclear Physics B, vol. 825, no. 3, pp. 426-443, 2010.

[12] A. H. Castro Neto, F. Guinea, N. M. R. Peres, K. S. Novoselov, and A. K. Geim, "The electronic properties of graphene," Reviews of Modern Physics, vol. 81, no. 1, pp. 109-162, 2009.

[13] A. V. Zhukov, R. Bouffanais, N. N. Konobeeva, and M. B. Belonenko, "On the electronic spectrum in curved graphene nanoribbons," JETP Letters, vol. 97, no. 7, pp. 400-403, 2013.

[14] M. Cvetic and G. W. Gibbons, "Graphene and the Zermelo optical metric of the BTZ black hole," Annals of Physics, vol. 327, no. 11, pp. 2617-2626, 2012.

[15] R. Kerner, G. G. Naumis, and W. A. Gómez-Arias, "Bending and flexural phonon scattering: generalized Dirac equation for an electron moving in curved graphene," Physica B: Condensed Matter, vol. 407, no. 12, pp. 2002-2008, 2012.

[16] F. de Juan, J. L. Mañes, and M. A. H. Vozmediano, "Gauge fields from strain in graphene," Physical Review B, vol. 87, Article ID 165131, 2013.

[17] A. Iorio, "Weyl-gauge symmetry of graphene," Annals of Physics, vol. 326, no. 5, pp. 1334-1353, 2011.

[18] R. E. Peierls, "Quelques proprietes typiques des corpses solides," Annales de l'Institut Henri Poincaré, vol. 5, pp. 177-222, 1935.

[19] L. D. Landau, "Zur Theorie der phasenumwandlungen II," Physikalische Zeitschrift der Sowjetunion, vol. 11, pp. 26-35, 1937.

[20] N. E. Firsova and Yu. A. Firsov, "A new loss mechanism in graphene nanoresonators due to the synthetic electric fields caused by inherent out-of-plane membrane corrugations," Journal of Physics D: Applied Physics, vol. 45, Article ID 435102, 2012.

[21] C. Liu, Z. Yu, D. Neff, A. Zhamu, and B. Z. Jang, "Graphenebased supercapacitor with an ultrahigh energy density," Nano Letters, vol. 10, no. 12, pp. 4863-4868, 2010.

[22] T. Ma, B. Li, and T. Chang, "Chirality- and curvature-dependent bending stiffness of single layer graphene," Applied Physics Letters, vol. 99, no. 20, Article ID 201901, 2011.

[23] M. B. Belonenko, N. G. Lebedev, N. N. Yanyushkina, A. V. Zhukov, and M. Paliy, "Electronic spectrum and tunneling current in curved graphene nanoribbons," Solid State Communications, vol. 151, no. 17, pp. 1147-1150, 2011.

[24] A. O. Barut and L. P. Singh, "Dirac equation in spatially nonflat Robertson-Walker space-times: II," International Journal of Modern Physics. D. Gravitation, Astrophysics, Cosmology, vol. 4, no. 4, pp. 479-490, 1995.

[25] M. Kober, B. Koch, and M. Bleicher, "The gravitational analogue to the hydrogen atom," European Journal of Physics, vol. 28, no. 3, article 465, 2007.

[26] N. Boulanger, P. Spindel, and F. Buisseret, "Bound states of Dirac particles in gravitational fields," Physical Review D, vol. D74, Article ID 125014, 2006.

[27] A. Sinha and R. Roychoudhury, "Dirac equation in $(1+$ 1)-dimensional curved space-time," International Journal of Theoretical Physics, vol. 33, no. 7, pp. 1511-1522, 1994. 
[28] Y. Sucu and N. Ünal, "Exact solution of Dirac equation in $2+1$ dimensional gravity," Journal of Mathematical Physics, vol. 48, no. 5, Article ID 052503, 2007.

[29] Ö. Açık, Ü. Ertem, M. Önder, and A. Verçin, "First-order symmetries of the Dirac equation in a curved background: a unified dynamical symmetry condition," Classical and Quantum Gravity, vol. 26, no. 7, Article ID 075001, 2009.

[30] A. Iorioa and G. Lambiase, “The Hawking-Unruh phenomenon on graphene," Physics Letters B, vol. 716, no. 2, pp. 334-337, 2012.

[31] M. J. Bueno, C. Furtado, and A. M. de M. Carvalho, "Landau levels in graphene layers with topological defects," The European Physical Journal B, vol. 85, p. 53, 2012.

[32] E. Perfetto, J. Gonzalez, F. Guinea, S. Belluci, and P. Onorato, "Quantum hall effect in carbon nanotubes and curved graphene strips," Physical Review B, vol. 76, no. 12, Article ID 125430, 2007.

[33] M. de Crombrugghe and V. Rittenberg, "Supersymmetric quantum mechanics," Annals of Physics, vol. 151, no. 1, pp. 99-126, 1983.

[34] A. Barducci, R. Casalbuoni, and L. Lusanna, "Supersymmetries and the pseudoclassical relativistic electron," Il Nuovo Cimento A Series 11, vol. 35, no. 3, pp. 377-399, 1976.

[35] C. Quesne, "Supersymmetry and the dirac oscillator," International Journal of Modern Physics A, vol. 06, p. 1567, 1991.

[36] A. Contreras-Astorga, D. J. Fernandez, and J. Negro, "Solutions of the Dirac equation in a magnetic field and intertwining operators," Symmetry, Integrability and Geometry: Methods and Applications, vol. 8, article 82, 2012.

[37] M. Salazar-Ramírez, D. Martínez, R. D. Mota, and V. D. Granados, "su $(1,1)$ algebraic approach of the Dirac equation with Coulomb-type scalar and vector potentials in D+1 dimensions," Europhysics Letters, vol. 95, no. 6, Article ID 60002, 2011.

[38] Ö. Yeşiltaş, "PT symmetric Hamiltonian model and Dirac equation in 1+1 dimensions," Journal of Physics A: Mathematical and Theoretical, vol. 46, no. 1, Article ID 015302, 2013.

[39] R. L. Hall and O. Yesiltas, "Supersymmetric analysis for the dirac equation with spin-symmetric and pseudo-spin-symmetric interactions," International Journal of Modern Physics E, vol. 19, no. 10, pp. 1923-1934, 2010.

[40] S. Bochner, "Über Sturm-Liouvillesche Polynomsysteme," Mathematische Zeitschrift, vol. 29, no. 1, pp. 730-736, 1929.

[41] D. Gómez-Ullate, N. Kamran, and R. Milson, "An extended class of orthogonal polynomials defined by a Sturm-Liouville problem," Journal of Mathematical Analysis and Applications, vol. 359, no. 1, pp. 352-367, 2009.

[42] C. Quesne, "Exceptional orthogonal polynomials, exactly solvable potentials and supersymmetry," Journal of Physics A: Mathematical and Theoretical, vol. 41, no. 39, Article ID 392001, 6 pages, 2008.

[43] C. Quesne, "Solvable rational potentials and exceptional orthogonal polynomials in supersymmetric quantum mechanics," SIGMA: Symmetry, Integrability and Geometry: Methods and Applications, vol. 5, article 084, 24 pages, 2009.

[44] B. Midya and B. Roy, "Exceptional orthogonal polynomials and exactly solvable potentials in position dependent mass Schrödinger Hamiltonians," Physics Letters A, vol. 373, no. 45, pp. 4117-4122, 2009.

[45] N. Rosen and P. M. Morse, "On the vibrations of polyatomic molecules," Physical Review, vol. 42, no. 2, pp. 210-217, 1932.

[46] C. B. C. Jasso and M. Kirchbach, "The trigonometric Rosen-Morse potential as a prime candidate for an effective QCD potential," AIP Conference Proceedings, vol. 857, article $275,2006$.
[47] C. B. Compean and M. Kirchbach, "Trigonometric quark confinement potential of QCD traits," European Physical Journal A, vol. 33, no. 1, pp. 1-4, 2007.

[48] B. Bagchi, "Position-dependent mass models and their nonlinear characterization," Journal of Physics A: Mathematical and Theoretical, vol. 40, no. 49, pp. F1041-F1045, 2007.

[49] C. A. de Carvalho and M. P. I. Filho, "Instantons and their molecules in QCD," Physical Review D, vol. 43, no. 10, pp. 34553464, 1991.

[50] T. Chen, S. Lin, and C. Jia, "Solutions of the Klein-Gordon equation with the improved Rosen-Morse potential energy model," European Physical Journal Plus, vol. 128, no. 7, pp. 1-6, 2013.

[51] A. Pnueli, "Spinors and scalars on Riemann surfaces," Journal of Physics A: Mathematical and General, vol. 27, no. 4, pp. 1345$1352,1994$.

[52] D.-H. Lee, "Surface states of topological insulators: the Dirac fermion in curved two-dimensional spaces," Physical Review Letters, vol. 130, no. 19, Article ID 196804, 2009.

[53] E. Abbena, S. Salamon, and A. Gray, Modern Differential Geometry of Curves and Surfaces with Mathematica, Chapman and Hall/CRC, 3rd edition, 2006.

[54] F. Cooper, A. Khare, and U. Sukhatme, Supersymmetry in Quantum Mechanics, World Scientific, River Edge, NJ, USA, 2001. 

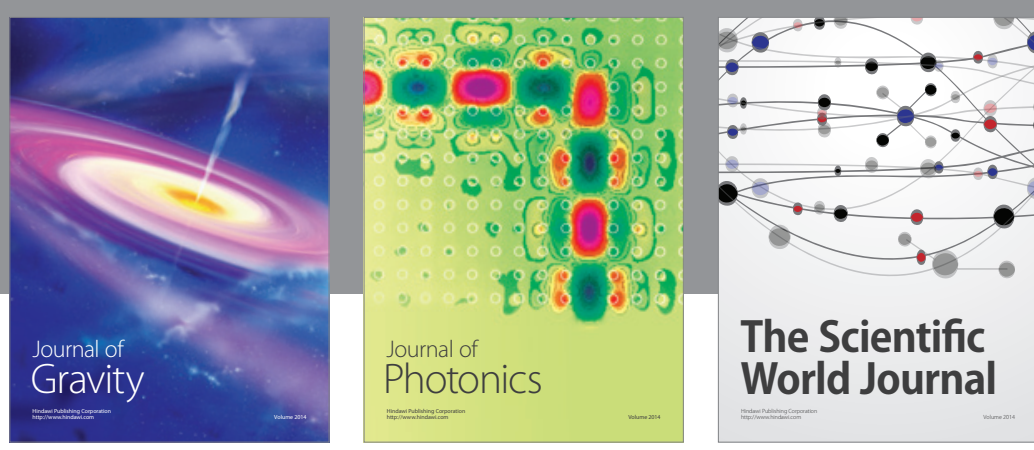

The Scientific World Journal
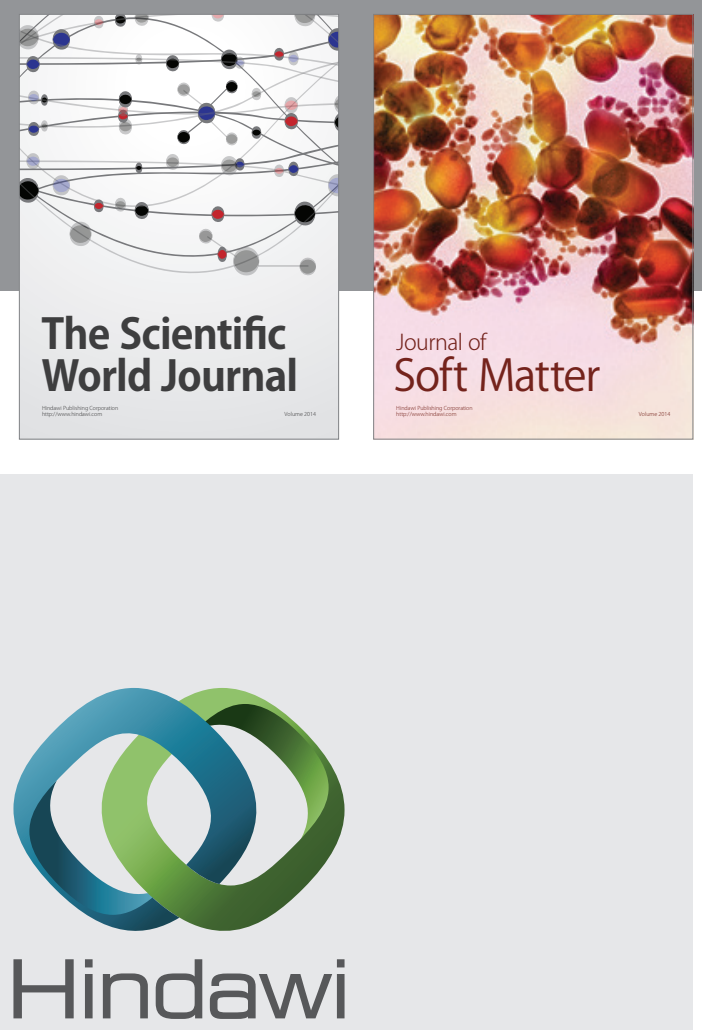

Submit your manuscripts at

http://www.hindawi.com

nternational Journal of

Statistical Mechanics
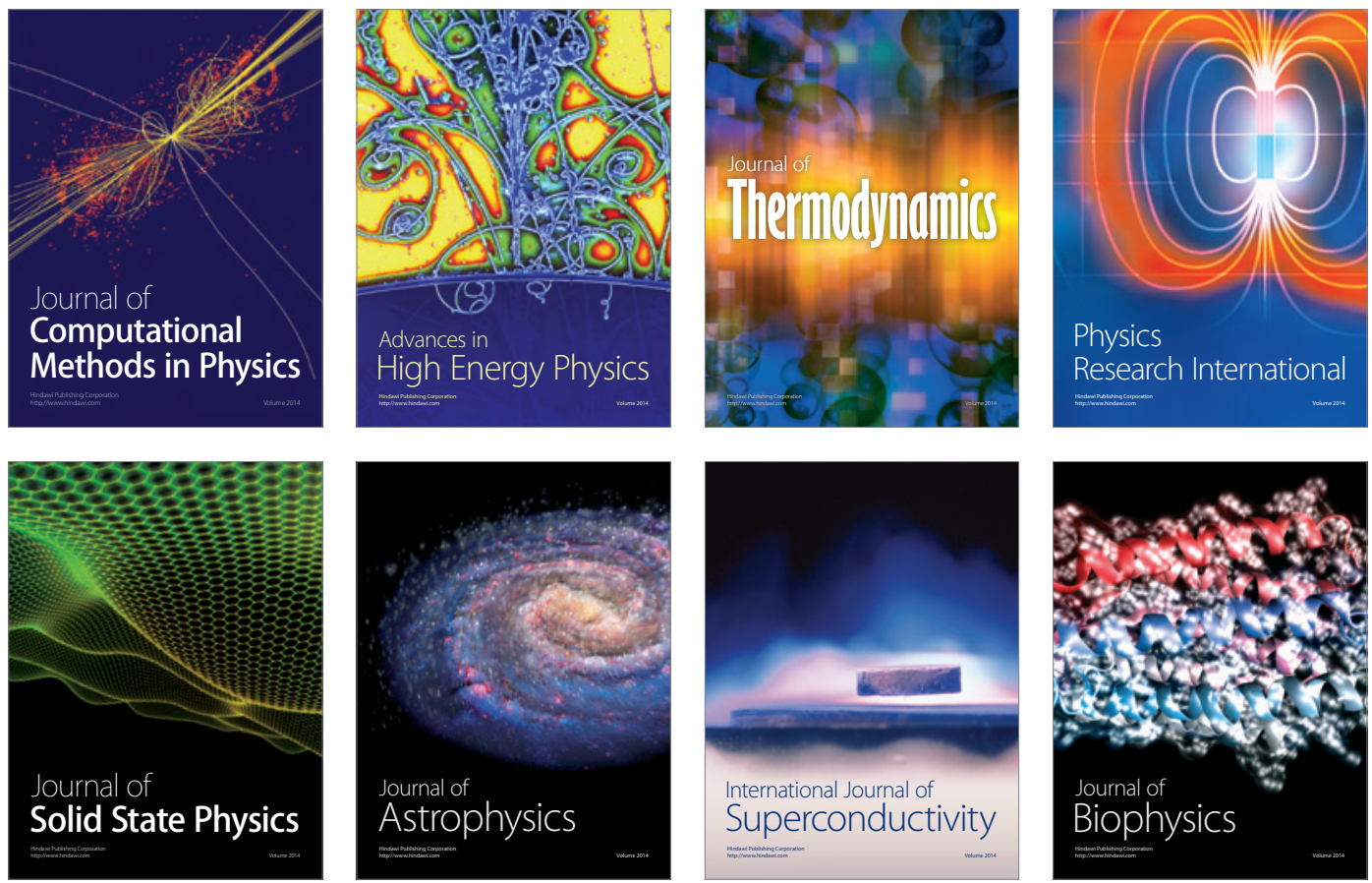
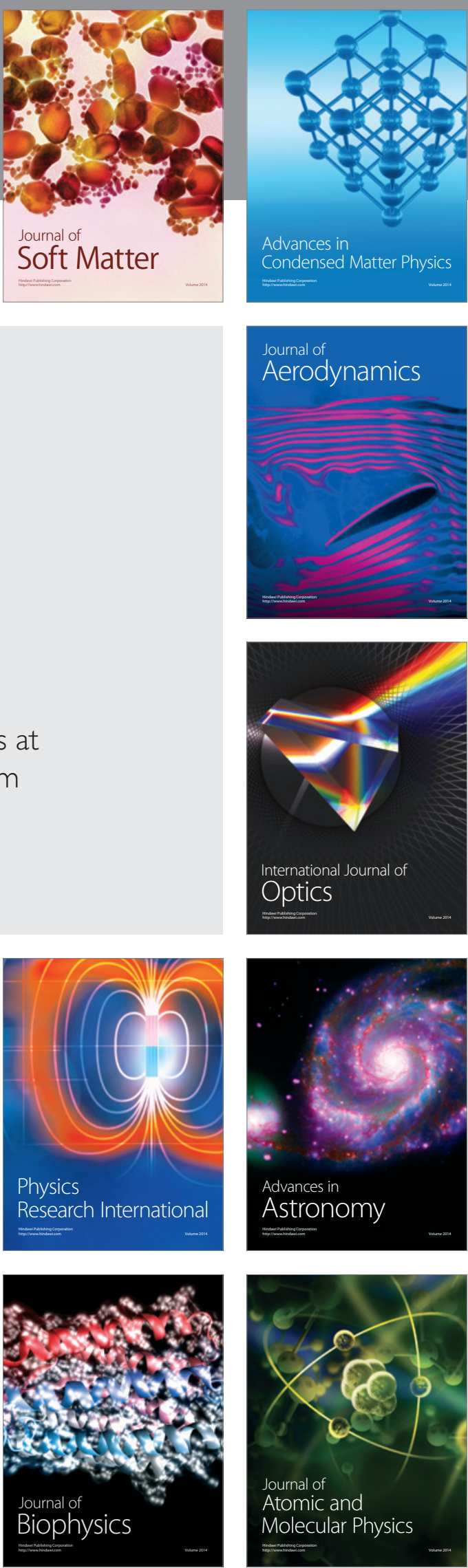